\title{
Sensing User Needs: Recognition Technologies and User Models for Adaptive User Interfaces
}

\author{
Barnabas Takacs ${ }^{1,2}$, Lajos Simon ${ }^{1}$, and Matthias Peissner ${ }^{3}$ \\ ${ }^{1}$ Semmelweis Medical University, \\ 1094 Budapest, Ferenc tér 15, Hungary \\ ${ }^{2}$ Technical University of Budapest, \\ 1111 Budapest, Magyar Tudosok Krt 2., Hungary \\ ${ }^{3}$ Fraunhofer IAO, Nobelstrasse 12, \\ 70569 Stuttgart, Germany \\ Btakacs@digitalcustom.com, simon@psych.sote.hu
}

\begin{abstract}
In this paper we introduce the notion of a Virtual User Lab that employs virtual reality tools to simulate End-Users in realistic application scenarios in order to help industrial designers and application developers to create and test adaptive interfaces that evolve as users' preferences and potential handicaps are discovered. We describe key elements of the VUL, discuss computer visionbased algorithms for facial information processing to understand user behavior and present an email-reading scenario to better highlight the system's adaptive capabilities and practical usability.
\end{abstract}

Keywords: User interface adaptation, facial information processing, virtual human interface.

\section{Introduction}

One of the key challenges in the process of developing adaptive user interfaces is not we not only need to sense the users' interaction and emerging patterns but further derive information about their intent and possibly internal state as an estimation of their likes and dislikes about the content being shown. This requires consistent interpretation of multiple sensory signals that are available in a given situation and interpret these signals in the context of the content being shown along with personalized user profiles. During the past decades, researchers have tried many input modalities to derive such information, but many of these failed due to the lack of a unified modeling and interpretation framework that has helped designers to test their products against life-like, but simulated situations.

To overcome this problem, we have designed a concept, called the Virtual User $L a b$ (VUL) that integrates the toolset of virtual reality and humanoid animation with an abstraction layer for various external sensor modalities, all delivering pieces of information about the behavior of a user while accessing the utilities provided by any specific application. This paper describes the architecture and sensory capabilities of the VUL and details how we process this information to help drive the underlying user interface adaptation processes, which is the main focus of our research. 
The classical treatment of Human Computer Interaction (HCI) considers the process of interaction between man and machine as somewhat unequal opponents [1]. The human element, i.e. the user, is involved in two basic processes: Perception and Control, while the computer's perspective on the other hand is frequently considered not as much along the lines of cognitive and intentional behavior, but as simple, low-level processes implemented in the form of agents. To make this pair equal the same modalities of communication readily available for people shall be implement for computers as well in order to give them a full set of senses complete with goals and cognition. This basic model of human computer interaction first assumes is that there are minimally two separate agents involved, one human and one machine. They are physically separated, but are able to exchange information through a number of information channels. On the side of the human user there are two basic processes involved, namely Perception and Control. The Perceptive process may be further divided into a) Human Input Channels (HIC) and b) Computer Output Media (COM) while similarly the Control process, comprises of c) Human Output Channels (HOC) and d) Computer Input Modalities (CIM). For a multi-modal HCI system that possesses an adaptive user interface that constantly improves during time, are especially interesting and challenging. Our goal is to create an intelligent user interface which is able to interpret the user's actions, decide for itself the best way to present information to the user, and cope with one of several input modalities selected by the user without the need to explicitly tell the computer.

User behaviors (like interest, boredom, excitement, confusion, fatigue, etc.) are accompanied by different patterns of facial expression, eye-gaze, head nod, hand movement, gestures and body posture. These so called surface level behaviors can be interpreted as indicators of "on-task" and "off-task" [3] in a general terminology and could be used as measures that help drive interface adaptation processes. On a greater scale, body postures convey specific meanings regarding the actions of the person in front of the output device (a TV or a computer screen), while the direction of eye gaze and head orientation, for example, are prime indicators of a person's focus of attention. More evidently spontaneous facial expressions and head nods are also good indicators of motivational and affective states in relation to the application. In particular, approving head nods, facial actions like smiles, tightening of the eyelids while concentrating, eyes widening, or raising eyebrows suggest interest and excitement (on-task). On the other hand, head shakes, the lowering of eyebrows, nose wrinkling and depressed lower lip corners indicate off-task behavior.

Clearly, these visually described behaviors often are easier to verify using other types, of specialized, non-visual sensors that are also more acceptable for End-Users protecting their privacy. Either case the output of these sensors must be interpreted in the context of an application itself and the system must form a consistent representation of the user's intention as it changes over time. Based on these observations we argue that virtual reality tools in combination with multi-modal sensory interfaces and advanced visual processing may be used to simulate and present such conditions and thus help industrial designers and application developers to better test how their designs will behave in realistic situations. This idea, what we call the Virtual User Lab, is the topic of the following section. 


\section{The Virtual User Lab}

The goal of the Virtual User Lab (VUL) is to create a virtual environment supporting industrial users in the development, simulation and evaluation of adaptive user interfaces for user groups with certain needs. Major features of the VUL focus on specific areas to $i$ ) to illustrate user profiles and their changes over time, $i i)$ to visualize possible user interface adaptations, iii) to simulate specific user interface designs and adaptation processes as experienced by a user with specific limitations, and $i v$ ) to create a pattern browser in order to provide designers and developers with an overview of design guidelines and an easy access to application specific design patterns which serve as reusable components for adaptive user interfaces. It is a multi-purpose key module of a more complex user interface system, which on one hand, serves as a virtual reality environment to access and visualize adaptation processes that take place in various application scenarios, and in another sense contains a virtual user that may be used to simulate user actions in response to a specific interface and verify adaptation processes during the design of such products or services. In order to keep its maximum flexibility the $V U L$ was also designed to interface to external programs and sensors that may be used to measure user reactions in-vivo and perform adaptations on the fly to prove the strength of different adaptation strategies. Finally, the same virtual user also serves as a tool to show designers how a person with impairments or limited physical capabilities would end up using their design and how adaptation processes would take place under those conditions. This is demonstrated in Fig 1.

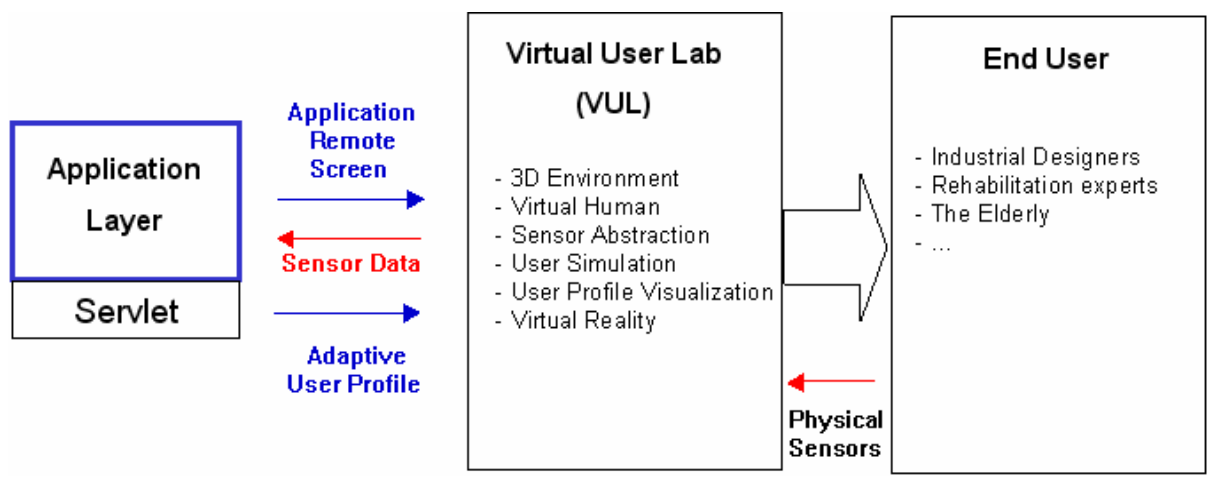

Fig. 1. Generic architecture and operation of the Virtual User Lab

\subsection{Physical Sensors vs. Data Abstraction Layer}

As in real-life application variety of external sensors may be used to indicate user presence, actions and intentions, which all provide similar types of information, but all differ in their physical manifestation and device characteristics, we first integrated an abstraction layer for an array of sensor types that generally correspond to capabilities of human senses, such as vision, hearing and tactile or motion-based feedback. The raw input data is processed by specialized algorithms is categorized into four distinct groups. We call this the Virtual Human Interface as demonstrated in Figure 2. 
The most important of these data streams are the images received from one or more video cameras. These are processed by a computer vision module, which primarily looks for faces, facial features, body gestures or other telltale signs of the user's presence, his or her actions or internal state. These algorithms correspond to a real web camera mounted on the top of the TV or alternatively viewing a virtual user in its own $3 \mathrm{D}$ environment and producing simulated virtual camera views for the adaptation algorithms to process or intelligent cameras and visual devices that provide already processed information on 3D and segmented components, such as the Kinect. The second set of algorithms employs signal processing and audio analysis to process data captured by the microphones. Tactile and Motion interfaces are implemented via touch screen and acceleration-based motion sensors, such as the Wii. Finally physical devices with no direct perceptual meaning are simply mapped further as raw data. Irrespective what channel the information is arriving from, the purpose of processing is to "recognize" and in some aspect "understand" the its meaning (e.g. to verify the presence of a face in front of the TV and analyze user's motion to arrive at inferences for adaptation purposes). The output of these mechanisms therefore can be mapped onto attributes of virtual markers that carry this information from the physical domain into an abstract symbolic representation suitable for the low-level reactive scripts and a high level Adaptation Engine (not described in this paper) to operate on.

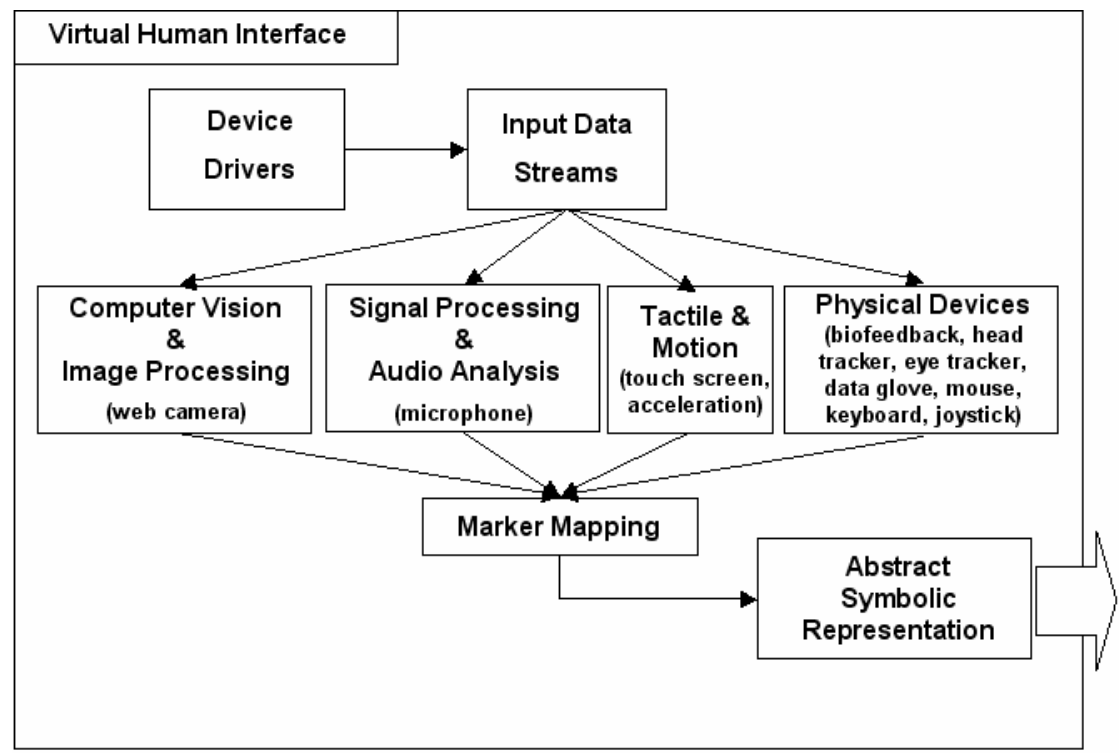

Fig. 2. Functional diagram of the Virtual Human Interface module responsible for processing sensory information

The module described above provides a real-time link between the end-user and the VUL via a symbolic representation of the outside world. This symbolic representation encodes events as they occur in the physical environment of the user and as an attempt to "understand" what is happening to him or her in the course of the 
interaction. Irrespective, however, of what the initial source of the data is, the essence of meaningful information it supports is encoded in the form of markers. Markers are dimensionless data holders with physical location and attributes in the synthetic 3D environment of the virtual human in the VUL. They carry unique hierarchical labels and attributes values encoding physical locations or abstract properties and they can be referred to by their names when describing high-level tasks in a rule-based behavior control system. They are used as input to the set of rules that control the adaptation algorithms of the entire framework.

To demonstrate how such abstraction is used to derive information about the user, in the next section we will discuss in more detail the visual processing capabilities of the VUL. Clearly these results maybe generalized to other classes of sensors as well.

\section{Facial Information Processing}

Most facial processing systems attempt to recognize user behavior, most particularly emotions on a person's face as a one-shot process using high level holistic models of how faces look or low-level feature extraction mechanisms that map directly onto emotions using neural networks or other types of statistical classification algorithms $[3,4]$. In the $V U L$ instead we created an algorithm that is based on forming a hypothesis with the help of low level cues and gradually refines that hypothesis using contextual and behavior rules. As a simple example, one may consider a simple face detector that is used the estimate the size of the head and in a correlated fashion the distance of the user's head from the screen. Such information could not only be useful for a HCI system to be able to adapt, e.g. the font size of an email reading application, but also provides clues whether the user can see what is on the screen well enough or not. Specifically, it has been suggested that a lean forward gesture may indicate the lack of clear visibility and the system could change the rendering of the messages in response.

Taking this idea further we can also analyze the facial regions as determined by the face detector and look for tell-tale signs of emotions, attention, etc. Region-based image processing is a basic technique identifying temporal events as they occur on the face. They can be executed in fast and more importantly in parallel to offer the possibility of collecting events that may (or may not!) signify a certain facial actions. Thus the VUL system views these events as "indicators" and resolves their potential meaning with the help of the emotion constraints known from psychology [5]. As facial tissue moves under the control of the muscles, darkened skin-lines in the form of edges frequently appear perpendicular to the direction of motion. These secondary facial features, such as sharply occurring temporary ridges due to the folding of skin, may be localized and their effect quantified. These features, called furrows and wrinkles, vary with each person, yet their very presence is strongly associated with the meaning of facial expressions. As an example, furrows in the upper facial area may be indicate that the user is thinking or trying to resolve/understand something very hard so we need to bring up a Help window. By using a face detector as mentioned above to locate the face and a rudimentary geometric model of facial regions, such as fore head, nose, mouth, etc., one may use localized image processing algorithms to measure the changes in these regions. In particular, these furrows and wrinkles are 
relatively easy to detect using real-time computer vision methods as they from rather characteristic horizontal and vertical edges. In our solution the image captured by the web-camera is first divided into facial regions using the eyes and nose detected and the sub-images corresponding to these facial regions of the upper-, middle and lower face, respectively are normalized before being passed on to the edge detection unit. The edge detection algorithm employs standard image processing filter operators and convolution masks in combination with morphological operations.

The operations carried out by these low-level image processing algorithms are demonstrated in Figure 3. Here the normalized images, the output of the binary operators, and the respective processing convolution kernels are shown. Different kernels are used to detect horizontal, vertical or diagonal lines. The outputs of these kernels are then combined into a single interpretation to provide a global measure describing the degree of furrows appearing in that portion of the image. On the left high gradient components of a face during a neutral and expressive state, respectively in the forehead and eye regions are shown, while on the right the output of the same operators for the mouth, chin and cheek regions are displayed. Besides edge detection, other real-time operators, such as optic flow and morphological filtering may also yield measures of widened eyes, a smile or open jaw. Figure 4 shows the typical output of these signals at the marker levels. While these signals individually are inherently noisy, together they provide a reliable measure that gradually enables the system to learn certain facial expressions and subsequently use them to better understand the users' needs.
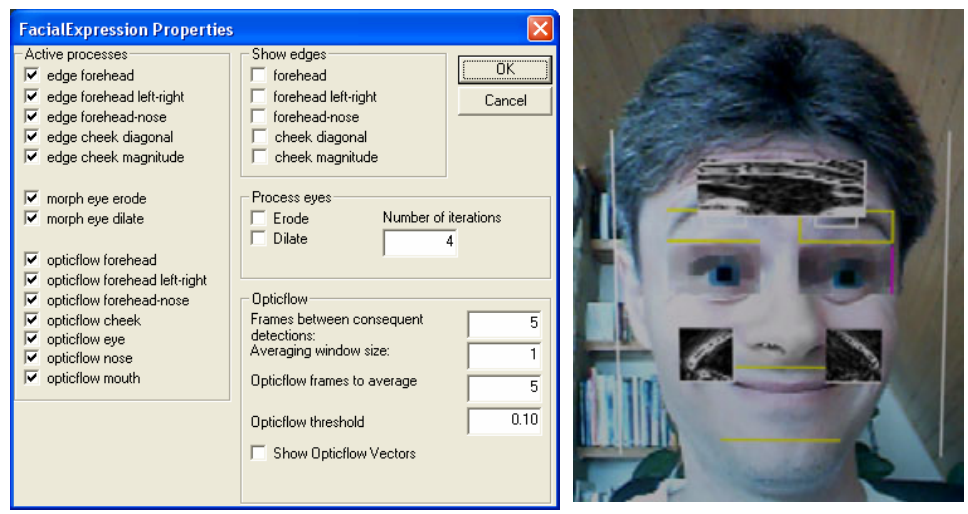

Fig. 3. Facial expression analysis using low-level image processing operators in specific regions of the face

To interpret these competing individual systems we map them on a circular, psychologically validated representation consistent with how humans express emotions during natural interaction. The set of competing rules attributed to each marker action shift the initial estimate of a neutral face in the direction of a specific emotion with a likelihood associated with the intensity of the facial event observed. The outcome of this process is a single estimate of the user's face indicating the most likely emotion he or she is in. 

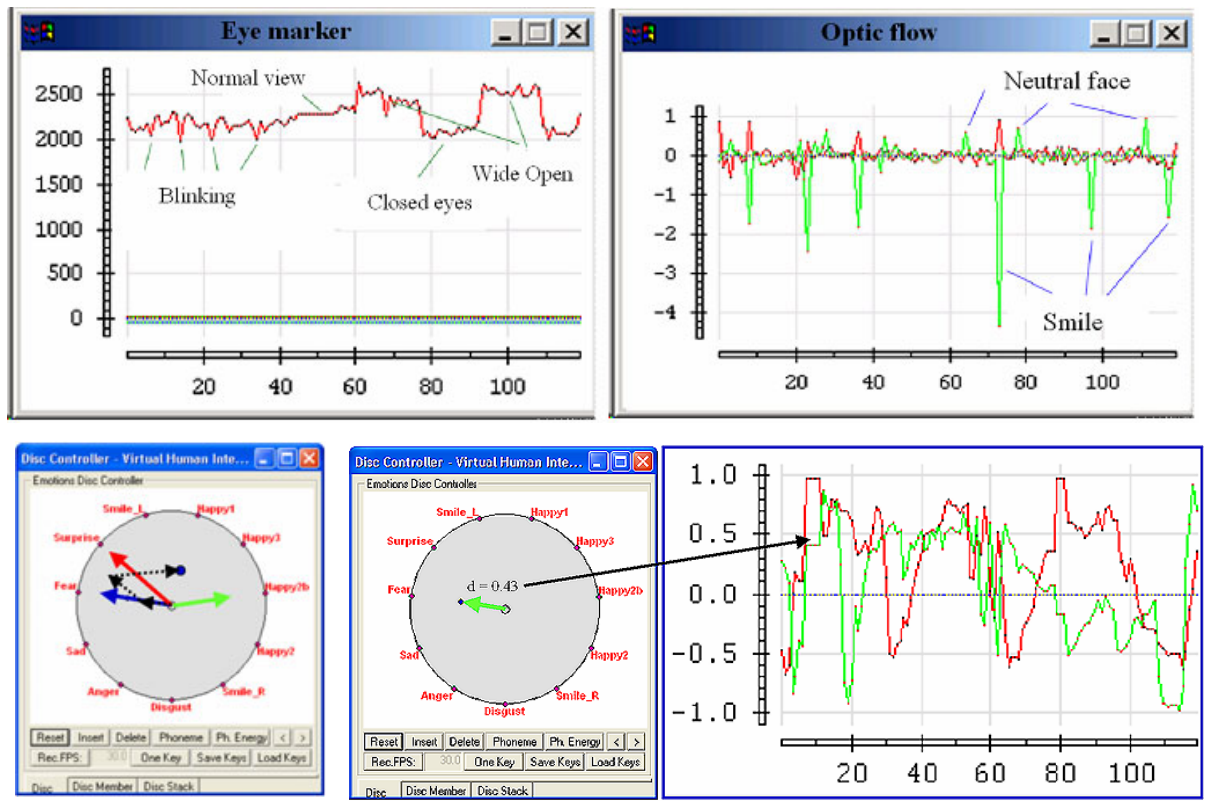

Fig. 4. Markers derived from consistent visual cues indicate the likelihood of local events on the face and in turn allow for a global interpretation of facial signals

\section{Practical Application Scenario}

The practical use of the Virtual User Lab hereby is demonstrated in a scenario designed for email reading applications using a remote adaptive server. In Figure 5 the main components of our system are shown. The GMail account the user is connected to renders the information contained in the email messages adaptively based on the user's behavior pattern. To achieve this goal the $V U L$ captures the remote email screen and displays it within one of two virtual environment, on a TV set in the virtual home and on the projector screen at the research lab, in this case. In both scenarios, the 3D environment allows the VUL to simulate different lighting and visibility conditions, as well as effect, like glare or even visual impairments of the End-User viewing this application. The user sees the email messages on a computer screen, or an industrial designer may experience it in a virtual reality headset (VR) for better immersion. The virtual environment is fully panoramic 360 view with many interactive elements most importantly a $3 D$ virtual remote controller, that may be used to control the remote application by pressing buttons. This virtual remote also allows the application to output physical infra red (IR) control signals and thus change channels on a physical device, as an example.

The Physical sensors attached to the VUL in this scenario are a web camera responsible for processing facial information, an ultrasonic distance sensor (as a second alternative to provide calibrated user distances and recognize gestures, such as leaning forward) an RFID reader to help identify different users and set preferences 
accordingly and finally a physical TV remote the signals of which are captured by the VUL and transmitted to the remote servlet simultaneously for adaptation purposes. In parallel to the physical signals, the facial information processor detects the face and outputs its estimated position and distance from the screen used again, to detect body gestures that may indicate poor visibility on the user's account.

The system is capable of recording and playing back all markers for testing, simulation and validation purposes. Designers interested in how their physical devices perform (e.g. the TV remote) under such conditions may create various visibility tests, realistically simulate visual impairments that rely on 3D distance information and rendering parameters and of course application adaptation rules on the server side may also be tested via simulated user interaction.

Application (Gmail)

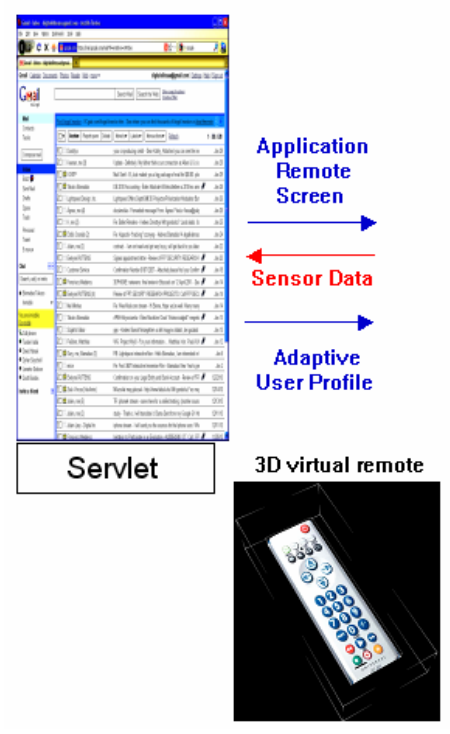

Virtual User Lab (VUL)

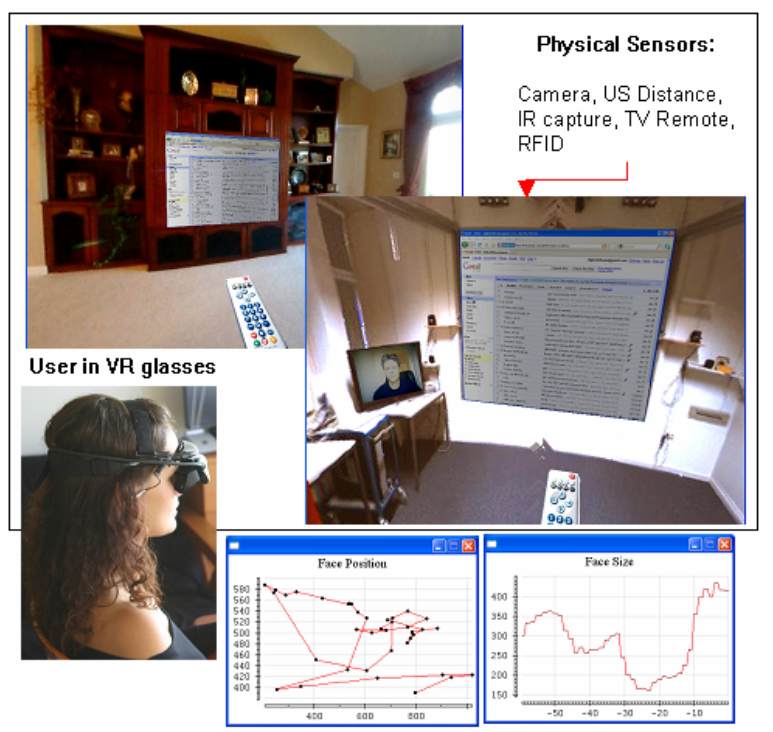

Fig. 5. Application scenario of the virtual user lab in a remote email reading case study using a NetTV and a virtual remote controller (see text)

\section{Summary}

In this paper we presented a novel approach to help user interface designers to create and test adaptive interfaces using a simulation tool, called the Virtual User Lab. Our approach combines the benefits of virtual reality to render a remote application in a virtual scene, change its viewing conditions and rendering quality both in terms of lighting and visibility factors or handicap as well as user behavior. The latter is measured with the help of a variety of local sensors that may be as simple as a web cam or more complex, like distance and motion sensors or even biofeedback. We have interfaced our solution with a client-server application model and demonstrated its use in a remote Email reading application. 


\section{References}

1. Schoemaker, L., et al.: Multimodal Integration for Advanced Multimedia Interfaces. In: ESPRIT III, Basic Research Project 8579 (2004)

2. Kapoor, A., Mota, S., Picard, R.W.: Towards a Learning Companion that Recognizes Affect. In: Proc. of Emotional and Intelligent Agents II: The Tangled Knot of Social Cognition, AAAI Fall Symposium (2001)

3. Bruce, V.: Face Recognition. In: Eyesenck, M.W. (ed.) Cognitive Psychology: An International Review (1992)

4. Kort, B., Reilly, R.: Analytical Models of Emotions, Learning and Relationships: Towards and Affect-sensitive Cognitive Machine. In: Proc. Intl. Conf. on Virtual Worlds and Simulation, San Antonio, TX (2002)

5. Schlossberg, H.: The Description of Facial Expressions in Terms of Two Dimensions. J. of Experimental Psychology 44(4) (1952) 